\title{
Considering People Living with Dementia When Designing Interfaces
}

\author{
Claire Ancient and Alice Good \\ School of Computing, University of Portsmouth \\ Buckingham Building, Lion Terrace, Portsmouth, UK, PO1 3HE \\ \{claire.ancient, alice.good\}@port.ac.uk
}

\begin{abstract}
Dementia is an escalating problem which is estimated to affect 35.6 million people worldwide. In an environment which is becoming increasingly dependent on technology, the interaction needs of people living with dementia is being ignored by interface designers. This paper aims to highlight the factors which should be considered when designing interfaces to be "dementiafriendly". The article draws on the limited previous research to suggest that interfaces need to consider two main factors: personalisation (which includes both accessibility and usability) and user acceptance (including the experience produced by the interfaces and barriers to technology adoption).
\end{abstract}

Keywords: Dementia, Interface Design, Personalisation, User Acceptance.

\section{Introduction}

The worldwide prevalence of dementia is rapidly increasing. In 2012, the World Health Organisation estimated a figure of 35.6 million people worldwide living with the condition, a number which is expected to rise by 7.7 million each year [1]. In the UK, 1 in 3 people over the age of 65 will develop a form of dementia [2].

Dementia is the term used for a collection of conditions including Alzheimer's disease and Vascular dementia. It is caused by damage to the brain, either due to diseases or a series of strokes. Alzheimer's disease is the most common form of dementia, affecting between $60 \%$ and $70 \%$ of diagnosed cases [1]. However, there are many rarer causes of dementia. Whilst each dementia-causing condition will have its own set of specific symptoms, people living with dementia will often experience short-term memory loss, problems with communication and difficulties with concentration [3].

The condition is progressive; therefore, the symptoms of dementia will get worse over time. In addition, every person living with dementia will experience the condition in their own way. This is dependent on a number of factors including (but not limited to): the type of dementia diagnosed, their personality, the medication prescribed and the support network around them [4]. 


\subsection{Importance of Technology}

Despite a lack of understanding and experience, previous research suggests older people are willing to utilise recent advances in technology, particularly when the device is considered beneficial [5]. In addition, research by Rosenberg et al. [6] suggests that people with dementia are able to utilise well-known technologies at a competent level. However, combining this reduced knowledge with interfaces that have been poorly designed and implemented can hinder the interaction and amplify pre-existing feelings of technical isolation and inadequacy $[7,8]$.

Technology, with special emphasis on assistive technology, when applied in an effective manner has the potential to improve the quality of life for both the person living with dementia and their caregiver allowing users the possibility of remaining in their own home longer; thus reducing the cost of residential care [5, 9-12]. Furthermore providing the opportunity to access therapeutic interventions in the users own time would provide people living with dementia an increased feeling of independence and improved levels of satisfaction [13]. However, there is often a stigma attached to the use of assistive devices [14]. Therefore, care needs to be taken to ensure the assistive device is appropriate for the needs of the person with dementia and mitigates any feelings of inadequacy. Examples of assistive technology include devices to promote safer walking, telecare to ensure safety within the home environment and memory aids to compensate for a declining cognitive ability [9].

It is important to note, that when assistive technology is introduced it should aim to augment the daily caring activities, rather than replace them totally. Whilst it is vital to find alternative methods to improve day-to-day life for both the person living with dementia and their caregiver, technology should not replace the vital human contact aspect of caring for someone [15].

By not considering the interaction needs of people living with dementia, interface designers may be isolating their intended users and hence negating the potential effectiveness of the developed technology. This could lead to prospective users disregarding a technology which could enhance their quality of life, enabling them to remain within the community and increase their safety.

\subsection{Objective}

Using secondary research in the form of a literature review, this paper aims to investigate the factors which need to be considered when designing interfaces for people living with dementia. By considering the interaction difficulties encountered due to dementia-related cognitive decline, designers can ensure technology is easily utilised by people living with the condition.

\section{Method}

In order to achieve the objectives of this paper, a literature search using the Discovery Service tool provided by the University of Portsmouth was conducted. This tool 
allowed the search of multiple library resources, including ScienceDirect, IEEE Xplore and the ACM Digital Library, to be explored simultaneously (for a full database listing visit: http: / / www . port.ac . uk/library/infores / discovery/).

Boolean logic was used to combine the keywords listed in table 1. This allowed the search results to be narrowed and ensured each of the title, abstract and subject terms were investigated for the inclusion of all keyword combinations. The Discovery search engine provides the functionality to search within the full text of a publication. However, in order to minimise the possible number of redundant results, it was decided not to utilise this functionality.

All the searches were limited to articles which have been published between January 2008 and December 2013. This ensured that only recent articles were obtained as part of the searching algorithm so as to only consider the most up-to-date knowledge and to obtain recommendations for the latest advances in technology, such as tablet computers.

Table 1. Search Keywords, chosen for their relevance to the topic reviewed

\begin{tabular}{|l|l|}
\hline Dementia & Interact* \\
\hline Comput* & Technolog* \\
\hline "User experience" & Experience \\
\hline Accessib* & Usab* \\
\hline "Design considerations" & "Design implications" \\
\hline Impair* & Mobile \\
\hline "Human factor" & "Human factors" \\
\hline HCI & "Interface design" \\
\hline Tablet & iPad \\
\hline Ubiquitous & Pervasive \\
\hline Interface & Accept* \\
\hline "User Acceptance" & Personal* \\
\hline Ergonom* & Adopt* \\
\hline
\end{tabular}

After the initial searches had been completed, the inclusion criteria described in section 2.1 was applied based upon the publication abstract and the subsequent duplicate articles removed.

\subsection{Inclusion Criteria}

The following inclusion criteria were applied to the abstracts of the articles found as a result of the initial searches: 
- Publications written in English

- Articles published in Academic Journals, Conference Proceedings and Books

- Publications where the focus is ensuring interfaces are suitable for people living with dementia (including accessibility, usability, user experience and user acceptance theory)

- Articles focusing on the technology rather than the design have been excluded from this current study, but have been reserved for future research.

\section{Results}

The results of this study showed that in the last six years minimal research has been carried out into the interaction needs of people living with dementia. The diagram in figure 1 shows the process carried out in order to reduce the number of possible articles from 1670 publications after the initial search to just 16 relevant studies.

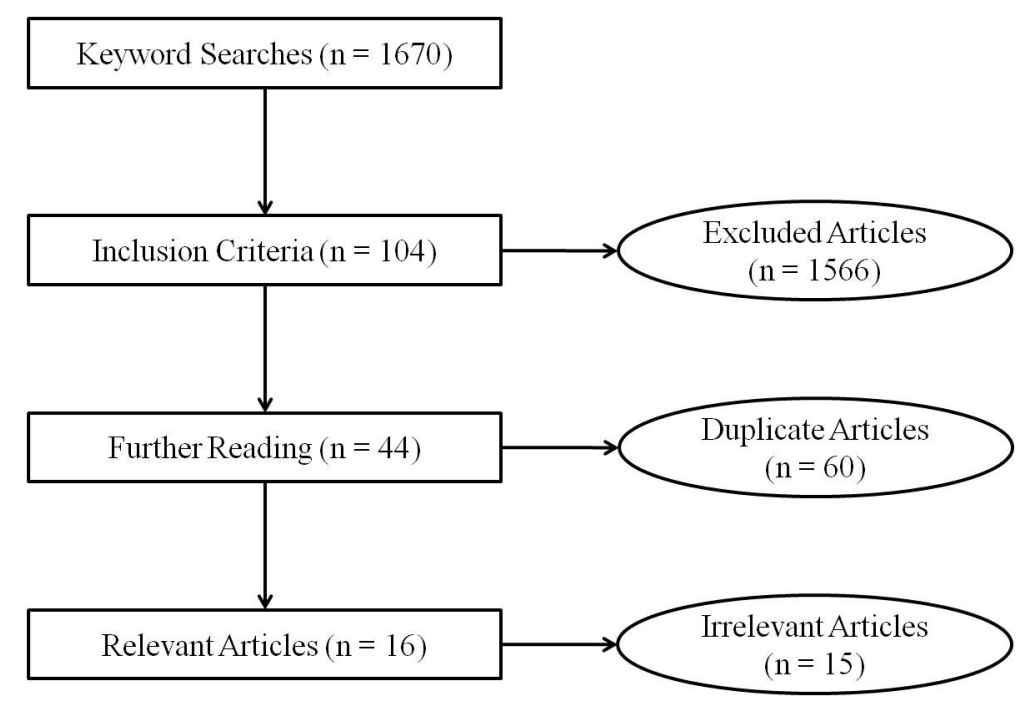

Fig. 1. Flowchart showing the process used to narrow the total number of articles

The initial searches yielded a total number of 1670 potential articles which could be relevant to the current study. The application of the inclusion criteria (described in the previous section) to the article abstracts reduced the number of publications to 104. Table 2 shows the total number of results for each search term together with the number of potentially useful results. 
Table 2. Search Results

\begin{tabular}{|c|c|c|}
\hline Search Terms & $\begin{array}{c}\text { Total } \\
\text { Number } \\
\text { of Results }\end{array}$ & $\begin{array}{l}\text { Useful } \\
\text { Results }\end{array}$ \\
\hline dementia AND interact* AND technolog* & 71 & 3 \\
\hline dementia AND interact* AND comput* & 78 & 5 \\
\hline dementia AND “user experience” & 0 & 0 \\
\hline dementia AND experience AND comput* & 44 & 2 \\
\hline dementia AND experience AND technolog* & 71 & 5 \\
\hline dementia AND accessib* AND comput* & 21 & 3 \\
\hline dementia AND accessib* AND technolog* & 24 & 2 \\
\hline dementia AND usab* AND comput* & 17 & 6 \\
\hline dementia AND usab* AND technolog* & 19 & 3 \\
\hline dementia AND "design considerations" & 4 & 0 \\
\hline dementia AND “design implications” & 0 & 0 \\
\hline dementia AND impair* AND technolog* & 189 & 5 \\
\hline dementia AND impair* AND comput* & 451 & 9 \\
\hline dementia AND mobile & 96 & 4 \\
\hline dementia AND ("human factor" OR "human factors") & 12 & 0 \\
\hline dementia AND hci & 6 & 1 \\
\hline dementia AND “interface design” & 1 & 1 \\
\hline dementia AND tablet AND comput* & 4 & 1 \\
\hline dementia AND tablet AND technolo* & 4 & 1 \\
\hline dementia AND iPad & 6 & 0 \\
\hline dementia AND ubiquitous AND comput* & 5 & 0 \\
\hline dementia AND ubiquitous AND technolog* & 3 & 1 \\
\hline dementia AND pervasive AND comput* & 9 & 3 \\
\hline dementia AND pervasive AND technolog* & 5 & 3 \\
\hline dementia AND interface AND comput* & 64 & 9 \\
\hline dementia AND interface AND technolog* & 20 & 5 \\
\hline dementia AND accept* AND technol* & 48 & 3 \\
\hline dementia AND accept* AND comput* & 46 & 3 \\
\hline dementia AND "user acceptance" & 3 & 1 \\
\hline dementia AND person* AND technolog* & 55 & 4 \\
\hline
\end{tabular}


Table 2. (Continued)

\begin{tabular}{|l|c|c|} 
dementia AND person* AND comput* & 64 & 6 \\
\hline dementia AND interact* AND person* & 159 & 2 \\
\hline dementia AND interface AND person* & 23 & 3 \\
\hline dementia AND ergonom* AND technolog* & 2 & 1 \\
\hline dementia AND ergonom* AND comput* & 6 & 3 \\
\hline dementia AND adopt* AND technolog* & 31 & 5 \\
\hline dementia AND adopt* AND comput* & 9 & 1 \\
\hline
\end{tabular}

Once the inclusion criteria had been applied, the duplicate articles were removed to produce a final list of 44 articles to be further investigated. These articles were read to ensure they were applicable to the current study at which point 15 articles were excluded.

The final number of articles suitable for inclusion in this study was 16 .

\section{Discussion of Relevant Articles}

In previous research, when designing interfaces three main factors were considered: accessibility, usability and user experience [16]. However, when designing for people living with dementia, two main ideas need to be considered holistically: personalisation and user acceptance, as shown by figure 2 .

\section{Interface}

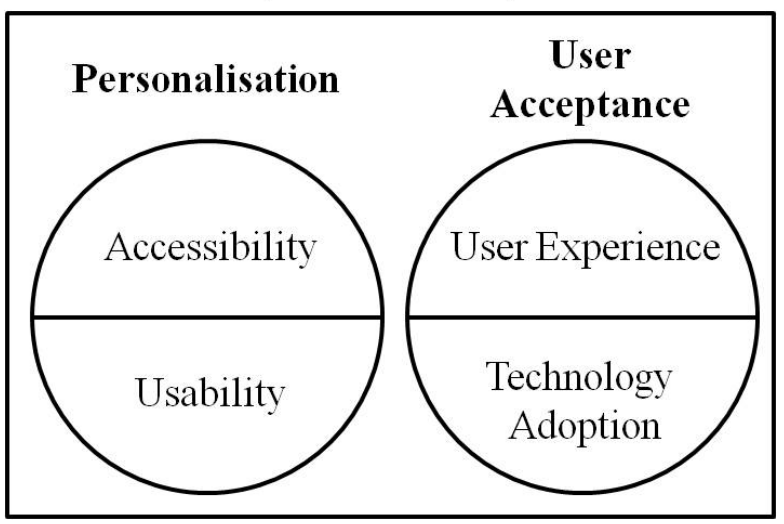

Fig. 2. Diagram to show the factors involved in interface design 


\subsection{Personalisation}

The concept of personalisation involves the adaption of the user interface in order to specifically meet the needs of the user. In this sense, personalisation would include features to ensure the interaction was both accessible and usable. Personalisation would be an ideal solution to catering for a wide variety of needs within a single group of users, making the technique appropriate for use with people living with dementia at all stages of the condition's progression [13].

A diagnosis of dementia does not automatically imply the person is unable to utilise technology to its full potential. Every person in different and should be treated as such. Any perceived interaction difficulties should be assessed on a case by case basis rather than making generalised assumptions about the state of a person's cognitive ability $[6,17]$.

The amount of personalisation an interface requires is a delicate process. Just like a human caregiver, it is important to ensure the right amount of support is provided in a timely manner. Both the system and the caregiver must provide the appropriate amount of assistance to empower the person living with dementia to complete the task without deskilling taking place [18]. As the person living with dementia's cognitive ability declines, the amount of support which is required from the system will increase. This leads to the requirement for the personalisation to be dynamic, and as such be able to adjust to the changing needs of the user over time [18].

In addition, the process of personalisation should tailor the interface to provide the necessary support to compensate for a person with dementia's declining abilities whilst maintaining the abilities which currently remain intact [5]. However, care needs to be taken that the interface is not simplified to a level which could patronise the person with dementia [10, 19]. Mihailidis et al. [13] argue that the interface should "start users with the most minimal possible starting point appropriate for their current level of abilities", reducing the likelihood of feeling overwhelmed. Rosenberg et al.'s study [5] showed that one concern of caregivers is that technology would be simplified to such an extent that it would "weaken a person's own abilities". By employing Mihailidis et al.'s approach to personalisation, the concerns of the caregivers would be allieviated.

The major downfall with the current user interface guidelines provided by the W3C WAI is the lack of flexibility and their apparent "one-size-fits-all" approach to design. The variability in the effects of dementia means that this approach to interface design will not work for this demographic. Flexibility is perceived as a crucial design requirement $[5,15]$ to ensure the interface is appropriate for the user, but could in turn become a hindrance should the person living with dementia be able to manually change the settings [5].

\subsection{User Acceptance}

User acceptance can be considered a critical success factor for projects to implement assistive technologies [18]. This concept can be split into two sections: user experience and technology adoption. These two aspects are inherently linked. If the 
interface does not provide a pleasurable interaction experience, the user will be less inclined to maintain usage. An engaging interaction experience will encourage users to adopt novel technologies, which could lead to acceptance of the device into everyday usage.

People living with dementia and their caregivers are more likely to adopt a novel technology if they perceive a need requiring support $[5,17,20]$. This increased interest remains regardless of whether the users encountered difficulties which required further explanations from supervisors [20]. However, Rosenberg et al. [5] are keen to point out that the potential users still need to experience the current need for the technology. In addition, the technology would need to seamlessly integrate within the person with dementia and their caregiver's existing habits [5, 15].

Previous experience of computing is also an important consideration for user acceptance. Research by deSant'Anna et al. [21] suggests that older users are reluctant to make use of IT through fear of causing irreparable damage to the system. This anxiety could jeopardise the effective use of the technology, negating its possible benefits [21]. In addition, the cognitive decline associated with dementia could diminish the person's ability to learn and retain the information required to operate the technology. This effect is enhanced when follow-ups and supported practice sessions are not implemented, increasing the likelihood of the technology being abandoned, despite an on-going need for continued use [22].

Both Aloulou et al. [18] and Starkhammar \& Nygard [22] argue that acceptance would be lower for people in the earlier stages of the dementia progression, when the perceived need is lower and higher in people whose abilities have significantly declined. Whilst people with a higher cognitive ability would find learning new interfaces easier, they may believe that they have no requirement for the system and therefore, may find the system (and the suggestion of implementation) both patronising and stigmatizing [10].

Self image plays an important part in the adoption and acceptance of assistive technology [5, 17]. Karlsson et al. [17] found that people with dementia utilised the devices in a fashion which complemented their own self images. If the use of the device did not fit in with their preconceived image they lost interest in using the technology.

Another important factor to consider when discussing technology adoption for people living with dementia is the influence of their caregivers. Research conducted by Karlsson et al. [17] suggests that spousal support for the integration of technology has an important influence over the person living with dementia's acceptance of novel devices. Whilst research carried out by both Hwang et al. [15] and McKenzie et al. [12] suggests that whilst caregivers are supportive of new technology aimed at reducing the burden of caring, they are reluctant to adjust their responsibility to the maintenance of the new systems. In addition, caregivers are reluctant to provide support for new technologies which were either inconvenient or gave their home an "institutional" feel [12]. By avoiding assistive technologies which have an institutional look, the caregivers may be helping the person with dementia to maintain their own self-image and esteem. 


\section{Conclusion}

In the last six years (January 2008 - December 2013) there has been minimal research focusing exclusively on ensuring interfaces are dementia-friendly. With the increasing number of people being diagnosed with the condition, this is a surprising conclusion. The increased prevalence of technology has the potential to provide multiple benefits, including enhanced independence and delaying the need for residential care. However, if the interfaces are not conducive to use by people living with dementia, the users may not be able to utilise assistive technologies to their full potential.

The research does, however, suggests that considering both personalisation and user acceptance is required to make an interface dementia-friendly. Due to the diverse nature of dementia, personalisation is particularly important for this demographic. By ensuring the interface adapts to the individual user's current circumstances, the interface would increase the accessibility and usability of the application. In addition, a dynamically adapting interface will allow the users to continue utilising the technology despite facing a progressive decline in their cognitive ability.

\subsection{Limitations}

This study has a number of limitations which will be addressed in future research.

Firstly, this study only includes papers which have been published in the last six years, between January 2008 and December 2013. As such, it will not include relevant papers which have been published in the preceding years. By including these articles increased awareness of the problems associated with utilising technology whilst living with the symptoms of dementia could be sought.

Secondly, people living with dementia also experience the natural effects of ageing [16]. Through the exclusion of articles which do not relate to dementia directly, this study has neglected to investigate important factors related to ageing which will also affect interaction with technology. The inclusion of research focused on the effects of ageing will serve to enhance the knowledge of impairments experienced by people living with a dementia diagnosis.

Finally, this study only included research where interaction with technology by people living with dementia is the main focus of the article. However, there are some studies where the main focus is on the use of technology and the design implications only briefly discussed. Through the inclusion of these studies, additional insights into interaction needs could be incorporated.

\subsection{Future Research}

The overall aim of the research, which includes this study, is to develop a set of guidelines with the goal of aiding interface designers to make interaction with technology dementia-friendly. This will allow the researchers to develop a smart application designed to be utilised by people living with dementia and their caregivers. The adherence to the developed set of guidelines will aim to ensure the application can be used to its full potential. 
Further relevant articles, which are outside the January 2008 to December 2013 publication date range will be obtained through the reference harvesting (or snowballing) technique. This will ensure that earlier research will be included within the developed guidelines. As part of this process, additional relevant search terms will be added to the list of keywords.

Finally, additional searches of the literature will be conducted to include additional keywords such as: older, elderly ageing and aged. These searches will inform the guidelines and allow them to take into account the natural effects of ageing. In addition, considering the interface needs of older people will aid interaction with the developed smart application for the caregiver of the person living with dementia.

\section{References}

1. World Health Organisation, http://www.who.int/mediacentre/factsheets/fs362/en/

2. Alzheimer's Society, http://www.alzheimers.org.uk/infographic

3. Alzheimer's Society, http://www.alzheimers.org.uk/site/scripts/ documents_info.php?documentID $=106$

4. Alzheimer's Society, http://www.alzheimers.org.uk/site/scripts/ documents_info.php?documentID $=133$

5. Rosenberg, L., Kottorp, A., Nygard, L.: Readiness for Technology Use with People with Dementia: The Perspectives of Significant Others. Journal of Applied Gerontology 31, 510-530 (2012)

6. Rosenberg, L., Kottorp, A., Winblad, B., Nygard, L.: Perceived Difficulty in Everyday Technology Use Among Older Adults With or Without Cognitive Deficits. Scandinavian Journal of Occupational Therapy 16, 216-226 (2009)

7. Chou, J., Hsiao, S.: A Usability Study on Human-Computer Interface for Middle-aged Learners. Computers in Human Behavior 23, 2040-2063 (2007)

8. Ziefle, M.: Information Presentation in Small Screen Devices: The Trade Off between Visual Density and Menu Foresight. Applied Ergonomics 41, 719-730 (2010)

9. Alzheimer's Society, http: / /www.alzheimers.org.uk/site/scripts/ documents_info.php?documentID=109

10. Nijhof, N., van Gemert-Pijnen, J., Burns, C., Seydel, E.: A Personal Assistant for Dementia to Stay at Home Safe at a Reduced Cost. Gerontechnology 11, 469-479 (2013)

11. Skillen, K., Chen, L., Nugent, C., Donnelly, M., Solheim, I.: A User Profile Ontology Based Approach for Assisting People with Dementia in Mobile Environments. In: 34th Annual International Conference of the IEEE EMBS, pp. 6390-6393 (2012)

12. McKenzie, B., Bowen, M., Keys, K., Bulat, T.: Safe Home Program: A Suite of Technologies to Support Extended Home Care of Persons with Dementia. American Journal of Alzheimer's Disease and Other Dementias 28, 348-354 (2013)

13. Mihailidis, A., Blunsden, S., Boger, J., Richards, B., Zutis, K., Young, L., Hoey, J.: Towards the Development of a Technology for Art Therapy and Dementia: Definition of Needs and Design Constraints. The Arts in Psychotherapy 37, 293-300 (2010)

14. Carrillo, M., Dishman, E., Plowman, T.: Everyday Technologies for Alzheimer's Disease Care: Research Findings, Directions and Challenges. Alzheimer's \& Dementia 5, 479-488 (2009) 
15. Hwang, A., Truong, K., Mihailidis, A.: Using Participatory Design to Determine the Needs of Informal Caregivers for Smart Home User Interfaces. In: 6th International Conference on Pervasive Computing Technologies for Healthcare (PervasiveHealth) and Workshops, pp. 41-48 (2012)

16. Ancient, C., Good, A.: Issues with Designing Dementia-Friendly Interfaces. In: Stephanidis, C. (ed.) HCII 2013, Part I. CCIS, vol. 373, pp. 192-196. Springer, Heidelberg (2013)

17. Karlsson, E., Axelsson, K., Zingmark, K., Savenstedt, S.: The Challenge of Coming to Terms with the Use of a New Digital Assistive Device: A Case Study of Two Persons with Mild Dementia. The Open Nursing Journal, 5, 102-110 (2011).

18. Aloulou, H., Mokhtari, M., Tiberghien, T., Biswas, J., Phua, C., Lin, J., Yap, P.: Deployment of Assistive Living Technology in a Nursing Home Environment: Methods and Lessons Learned. BMC Medical Informatics and Decision Making 13 (2013)

19. Mahmud, N., Vogt, J., Luyten, K., Slegers, K., Van den Bergh, J., Coninx, K.: Dazed and Confused Considered Normal: An Approach to Create Interactive Systems for People Living with Dementia. In: Bernhaupt, R., Forbrig, P., Gulliksen, J., Lárusdóttir, M. (eds.) HCSE 2010. LNCS, vol. 6409, pp. 119-134. Springer, Heidelberg (2010)

20. Gonzalez-Palau, F., Franco, M., Toribio, J., Losada, R., Parra, E., Bamidis, P.: Designing a Computer-based Rehabilitation Solution for Older Adults: The Importance of Testing Usability. PsychNology Journal 11, 119-136 (2013)

21. de Sant'Anna, M., Vallet, C., Kadouche, R., Stefanucci, D., Tomascakova, A., Morat, B., Rigaud, A.-S.: Computer Accessibility for Individuals Suffering from Mild to Moderate Alzheimer's Disease. European Geriatric Medicine 1, 186-192 (2010)

22. Starkhammar, S., Nygard, L.: Using a Timer Device for the Stove: Experiences of Older Adults with Memory Impairment or Dementia and their Families. Technology and Disability 20, 179-191 (2008) 